Pathologe 2016 37 (Suppl 2):S173-S174 DOI 10.1007/s00292-016-0215-3

Online publiziert: 14. September 2016 (c) Der/die Autor(en) 2016. Dieser Artikel ist eine Open-Access-Publikation.

CrossMark

\author{
J. Pencik \\ Medizinische Universität Wien, Wien, Österreich
}

\title{
STAT3-reguliertes ARF hemmt Metastasierung von Prostatakrebs
}

aggressiveren PTEN-defizienten Prostatatumoren führt. Zusätzlich führte die In-vivo-Inhibierung von JAK1/JAK2Ruxolitinib zu vergrößertem Wachstum und reduzierter STAT3/p14 ${ }^{\mathrm{ARF}}$-Expression humaner Prostatekrebszellen in Xenograft-Modellsystemen.

Die klinische Relevanz dieser Erkenntnisse ist von äußerster Wichtigkeit, da wir anhand der Analyse von über 200 Patientenproben zeigen konnten, dass der Verlust der STAT3- oder p14 ${ }^{\mathrm{ARF}}$ Expression mit einer schlechteren Überlebensrate korreliert. Daher konnten wir beide Faktoren als neue prognostische Marker identifizieren.

Zusätzlich korrelierte der Verlust der STAT3- oder p14 ${ }^{\mathrm{ARF}}$-Expression signifikant mit einem erhöhten Gleason-Score, verfrühtem biochemischem Wiederauf- treten sowie verstärkter Metastasierung. Weiter zeigen wir Mutationen in primären Prostatatumoren und, noch häufiger, genetische Verluste von STAT3 und ARF in metastasierenden Prostatatumoren.

Zusammenfassend kann die Analyse von STAT3- und ARF-Expression in Tumoren Patienten in Hoch- und Niedrigrisikogruppen aufteilen, was eine aktive Überwachung des Krankheitsverlaufs ermöglicht. Diese Ergebnisse sind nicht nur wichtig für das klinische Management von Prostatakrebspatienten, sondern auch für andere Tumorarten. Dadurch können die behandelnden Ärzte früher entscheiden, welche weiteren Therapieschritte notwendig sind.

Erstaunlicherweise fanden wir, dass die IL-6/STAT3-Signalkaskade den Verlust von Phosphatase-and-tensin-homolog(PTEN)-induzierter Seneszenz via $A R F$ vermittelt (• Abb. 1). Weiter konnten wir erstmalig ARF als direktes Target-Gen von STAT3 identifizieren. Mithilfe prostataspezifischer Mausmodelle zeigten wir, dass der genetische Verlust von STAT3 zu aggressiven und metastasierenden PK führt. Dies kann mit der fehlenden STAT3-induzierten Unterdrückung von ARF erklärt werden. Diese Ergebnisse sind äußerst wichtig, da STAT3 in diesem Kontext als Onkogen bekannt ist. STAT3 wird hauptsächlich vom Zytokin IL-6 aktiviert. Wir konnten zeigen, dass auch die genetische Deletion von IL-6 im Mausmodell zu

Dieser Beitrag basiert auf der Original publikation Pencik J, Schlederer M, Gruber W et al (2015) STAT3 regulated ARF suppresses prostate cancer metastasis. Nat Commun 6:7736
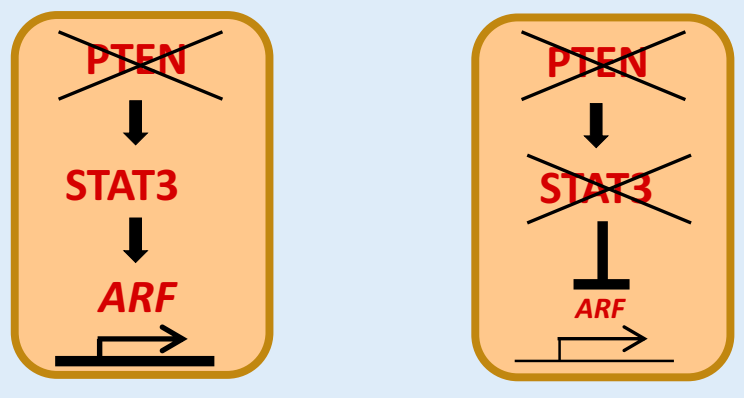

resultiert in

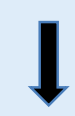

Seneszenz in

Primärtumoren
Abb. $1<$ Der Verlust des STAT3-ARFSignalwegs fördert die Metastasierung von Prostatakrebs 


\section{Korrespondenzadresse}

\section{Dr. J. Pencik}

Medizinische Universität Wien

Spitalgasse 23, 1090 Wien, Österreich

jan.pencik@meduniwien.ac.at

Open access funding provided by Medical University of Vienna.

\section{Einhaltung ethischer Richtlinien}

Interessenkonflikt. J. Pencik gibt an, dass kein Interessenkonflikt besteht.

Alle angewandten Verfahren stehen im Einklang mit den ethischen Normen der verantwortlichen Kommission für Forschung am Menschen (institutionell und national) und mit der Deklaration von Helsinki von 1975 in der revidierten Fassung von 2008. Alle Patienten wurden erst nach erfolgter Aufklärung und Einwilligung in die Studie eingeschlossen. Hiermit bestätige ich als korrespondierender Autor, dass alle nationalen Richtlinien zur Haltung und zum Umgang mit Labortieren eingehalten wurden und die notwendigen Zustimmungen der zuständigen Behörden vorliegen.

The supplement containing this article is not sponsored by industry.

Open Access. Dieser Artikel wird unter der Creative Commons Namensnennung 4.0 International Lizenz (http://creativecommons.org/licenses/by/4.0/deed. de) veröffentlicht, welche die Nutzung, Vervielfältigung, Bearbeitung, Verbreitung und Wiedergabe in jeglichem Medium und Format erlaubt, sofern Sie den/die ursprünglichen Autor(en) und die Quelle ordnungsgemäß nennen, einen Linkzur Creative Commons Lizenz beifügen und angeben, ob Änderungen vorgenommen wurden. 